Supporting Information:

\title{
Monolayer-Protected Nanoparticle Film Assemblies as Platforms for Controlling Interfacial and Adsorption Properties in Protein Monolayer Electrochemistry
}

\author{
Andrew F. Loftus, Katelyn P. Reighard, Susanna A. Kapourales, and Michael C. Leopold* \\ Department of Chemistry, Gottwald Center for the Sciences, University of Richmond \\ Richmond, VA 23173
}

\section{Contents:}

- Procedural details for assembly of metal-linked, ester-coupled, and dithiol-linked MPC films.

TEM histogram analysis and representative images for the different-sized nanoparticles.

Double layer capacitance of carboxylate-metal ion-carboxylate linked MPC assemblies tracked over multiple dipping cycles

MPC film assembly, double layer capacitance optimization - core size

MPC film assembly, double layer capacitance optimization - ligand chainlength

Contact angle measurements on a range of different modified substrates

Characterization of MUA-capped, hydrophilic MPCs (UV-Vis, ${ }^{1} \mathrm{H}$ NMR, and TEM)

Atomic force microscopy of evaporated gold substrates with and without MPC film assembly

Schematic of inverted electrochemical cell 


\section{Film Assembly Details:}

Carboxylic acid-Zn ${ }^{2+}$ linked MPC Film Assembly. An initial SAM of 11-mercaptoundecanoic acid (MUA) was formed on the electrochemically cleaned evaporated gold mounted in the inverted cell. The MUA SAM was washed with copious amounts of ethanol before being submerged with a $1 \mathrm{mM} \mathrm{KOH}$ ethanol solution for 10-15 minutes in order to deprotonate the carboxylic acid end groups. The cell was then filled with a solution of $0.2 \mathrm{M} \mathrm{Zn}\left(\mathrm{NO}_{3}\right)_{2}$ in ethanol and bubbled with nitrogen for 10-20 minutes to create a stirring effect within the cell. The cell was then rinsed several times with ethanol and refilled with a solution of carboxylic acid functionalized MPCs $(1 \mathrm{mg} / \mathrm{mL})$, previously prepared with a place-exchange reaction between plain MPCs and MUA ligands. Prior to using the MUA-MPCs in the film assembly, 10-15 $\mu \mathrm{L}$ of $0.2 \mathrm{M} \mathrm{KOH}$ in ethanol was added to the MUA-MPC solution to facilitate carboxylate-metal ioncarboxylate linking bridges. Again, this solution was bubbled with nitrogen (stirred) for 1 hour before the cell was thoroughly rinsed again with ethanol and the entire process repeated. These two steps, exposure to $\mathrm{Zn}\left(\mathrm{NO}_{3}\right)_{2}$ and MUA-MPC solutions were repeated until the desired film growth was achieved and the process capped with a final layer of MPCs.

Ester-Coupled MPC Film Assembly. For this study, an initial SAM of 11-mercaptoundecanoic acid (MUA) was formed on an electrochemically cleaned evaporated gold substrate mounted in an inverted electrochemical cell via overnight exposure of the substrate to a MUA ethanol solution. The MUA SAM was washed with copious amounts of ethanol and exposed to an ethanolic solution of 16-mercaptoundecanoic acid (MHDA) for one hour before being rinsed with ethanol. The resulting mixed SAM was then rinsed with dichloromethane $\left(\mathrm{CH}_{2} \mathrm{Cl}_{2}\right)$ before being submerged in a solution of MPCs modified with 11-mercaptoundecanol (MUD-MPC at 1mg/mL) in $\mathrm{CH}_{2} \mathrm{Cl}_{2}$ that also contained ester coupling reagent cyclohexylcarbodiimide (DCC) and catalyst 4-(dimethyl-amino)pyridine (DMAP). This mixture was bubbled with nitrogen for 1 hour (stirred) prior to removing the substrate and washing it with $\mathrm{CH}_{2} \mathrm{Cl}_{2}$. The substrate was then immersed in a solution of MPCs modified with 11-mercaptoundecanoic acid (MUA-MPC at $1 \mathrm{mg} / \mathrm{mL}$ ) in $\mathrm{CH}_{2} \mathrm{Cl}_{2}$ with added DCC and DMAP. This mixture was bubbled with nitrogen for 1 hour (stirred) prior to removing the substrate and washing it with $\mathrm{CH}_{2} \mathrm{Cl}_{2}$. This procedure was then repeated with alternating exposure to MUD-MPC and MUA-MPC solutions with added DCC and DMAP until the desired growth was achieved and the process capped with a final layer of MPCs. Both the MUA-MPC and MUD-MPC were prepared via place-exchange reactions prior to growth assembly.

Dithiol-Linked MPC Film Assembly. An initial SAM of 6-mercaptohexane (C6) was formed on the electrochemically cleaned evaporated gold mounted in the inverted cell. The cell was then rinsed with ethanol and exposed to an ethanol solution of $5 \mathrm{mM}$ 1,9-nonanedithiol (NDT) for 1 hour. NDT should effectively displace a portion of the hexanthiolates comprising the SAM and an exposed thiol group at the SAM/solution interface that is used to anchor a layer of MPCs. The mixed SAM was then rinsed with ethanol and the cell immediately filled with unfunctionalized MPC solution, normally C6-MPCs $(1 \mathrm{mg} / \mathrm{mL})$ in dichloromethane $\left(\mathrm{CH}_{2} \mathrm{Cl}_{2}\right)$. The solution in the cell was then bubbled with nitrogen (stirred) for 40 minutes, rinsed with dichloromethane. The cell is then filled with a $5 \mathrm{mM}$ NDT in $\mathrm{CH}_{2} \mathrm{Cl}_{2}$ (the same solvent as the MPCs) and bubbled for with nitrogen for another 20 minutes before being emptied and washed with $\mathrm{CH}_{2} \mathrm{Cl}_{2}$. This procedure was then repeated with alternating exposure to MPC and NDT solutions until the desired growth was achieved and the process capped with a final layer of MPCs (see Experimental Details and text for procedures related to fifth layer MPCs). 

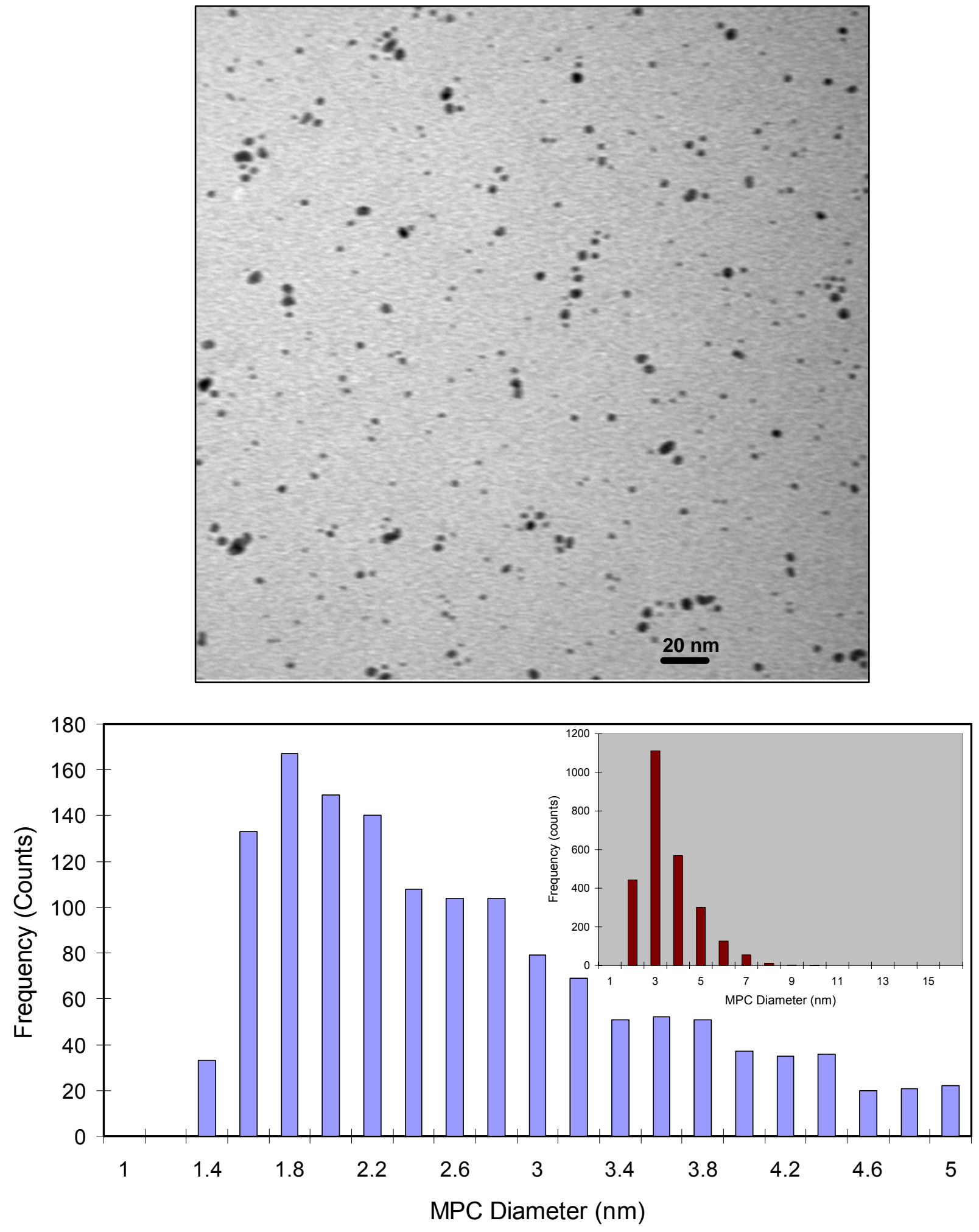

Figure SI-1. Typical TEM image (top) of nanoparticles with an average composition of $\mathrm{Au}_{807} \mathrm{C}_{163}$. Core size histogram (bottom) from image analysis of multiple TEM images of as prepared $\mathrm{Au}_{807} \mathrm{C6}_{163}$ samples indicate an average diameter of $2.85 \pm 1.35 \mathbf{~ n m}$. Inset: Histogram with larger bin sizes determined from the same set of images. 


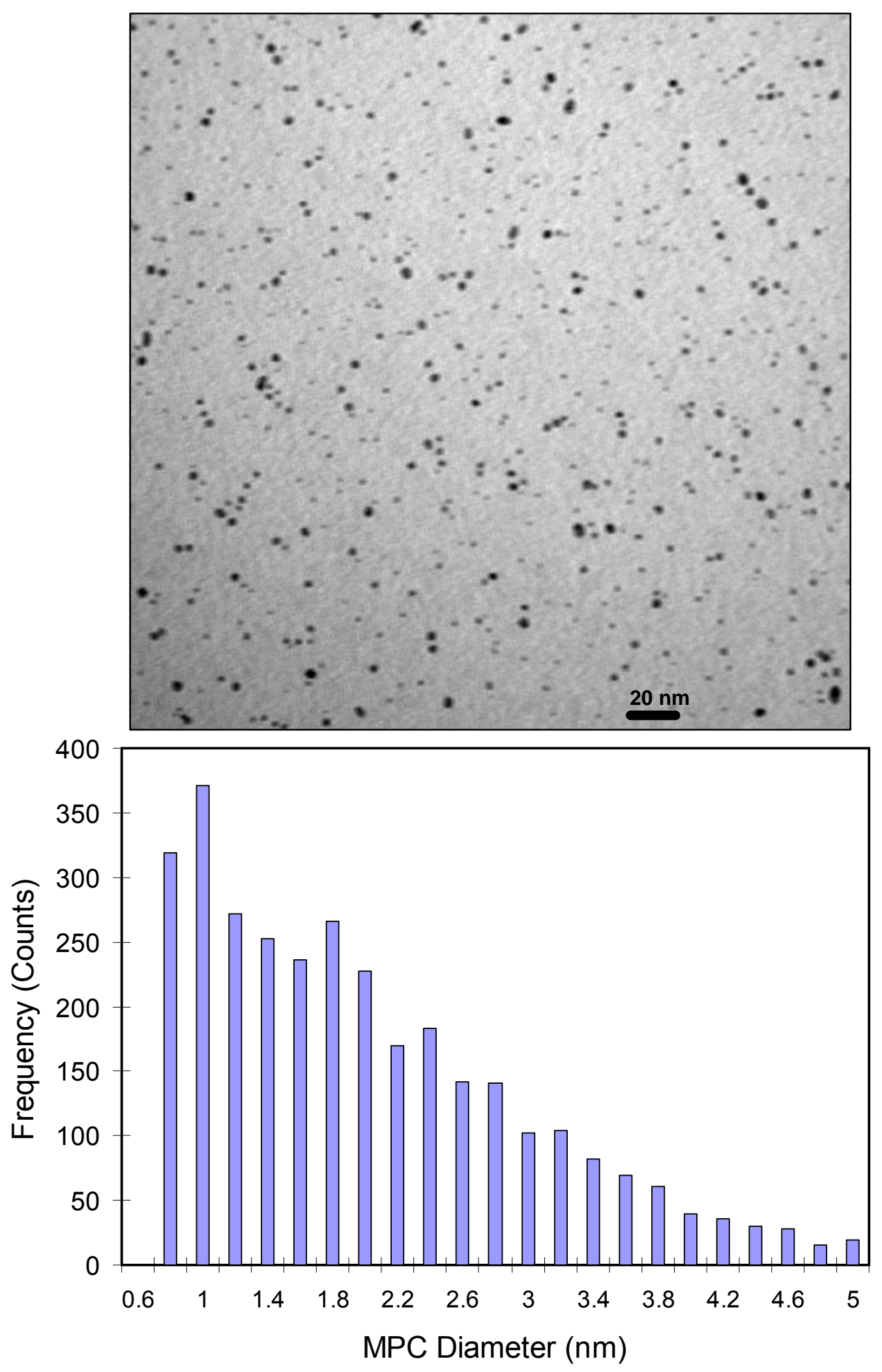

Figure SI-2. Typical TEM image (top) of nanoparticles with an average composition of $\mathrm{Au}_{225} \mathrm{C}_{75}$. Core size histogram (bottom) from image analysis of multiple TEM images of as prepared $\mathrm{Au}_{225} \mathrm{C}_{75}$ samples indicate an average diameter of $2.03 \pm 0.95 \mathbf{~ n m}$. 

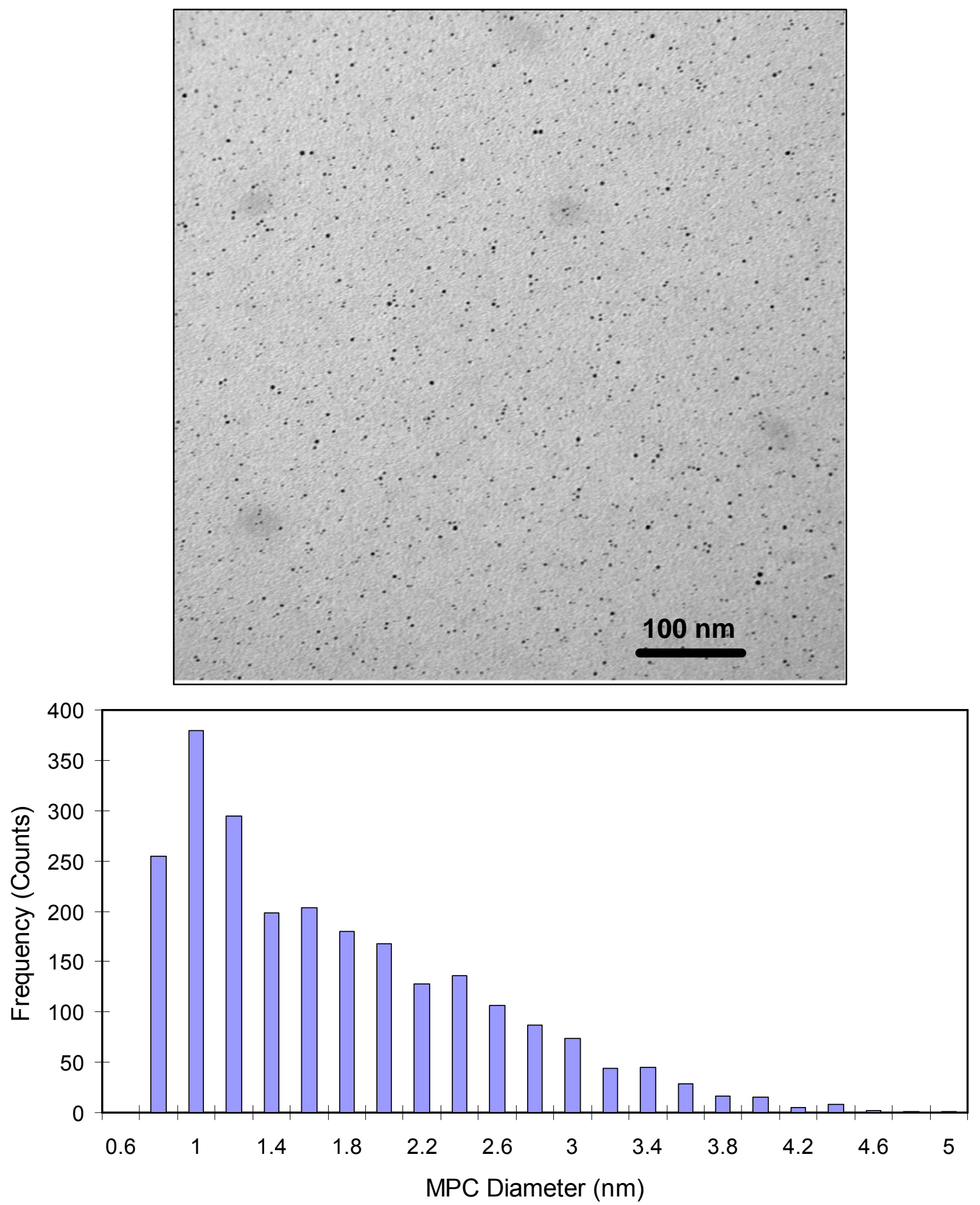

Figure SI-3. Typical TEM image (top) of nanoparticles with an average composition of $\mathrm{Au}_{140} \mathrm{C}_{53}$. Core size histogram (bottom) from image analysis of multiple TEM images of as prepared $\mathrm{Au}_{140} \mathrm{C}_{53}$ samples indicate an average diameter of $1.76 \pm 0.77 \mathbf{~ n m}$. 


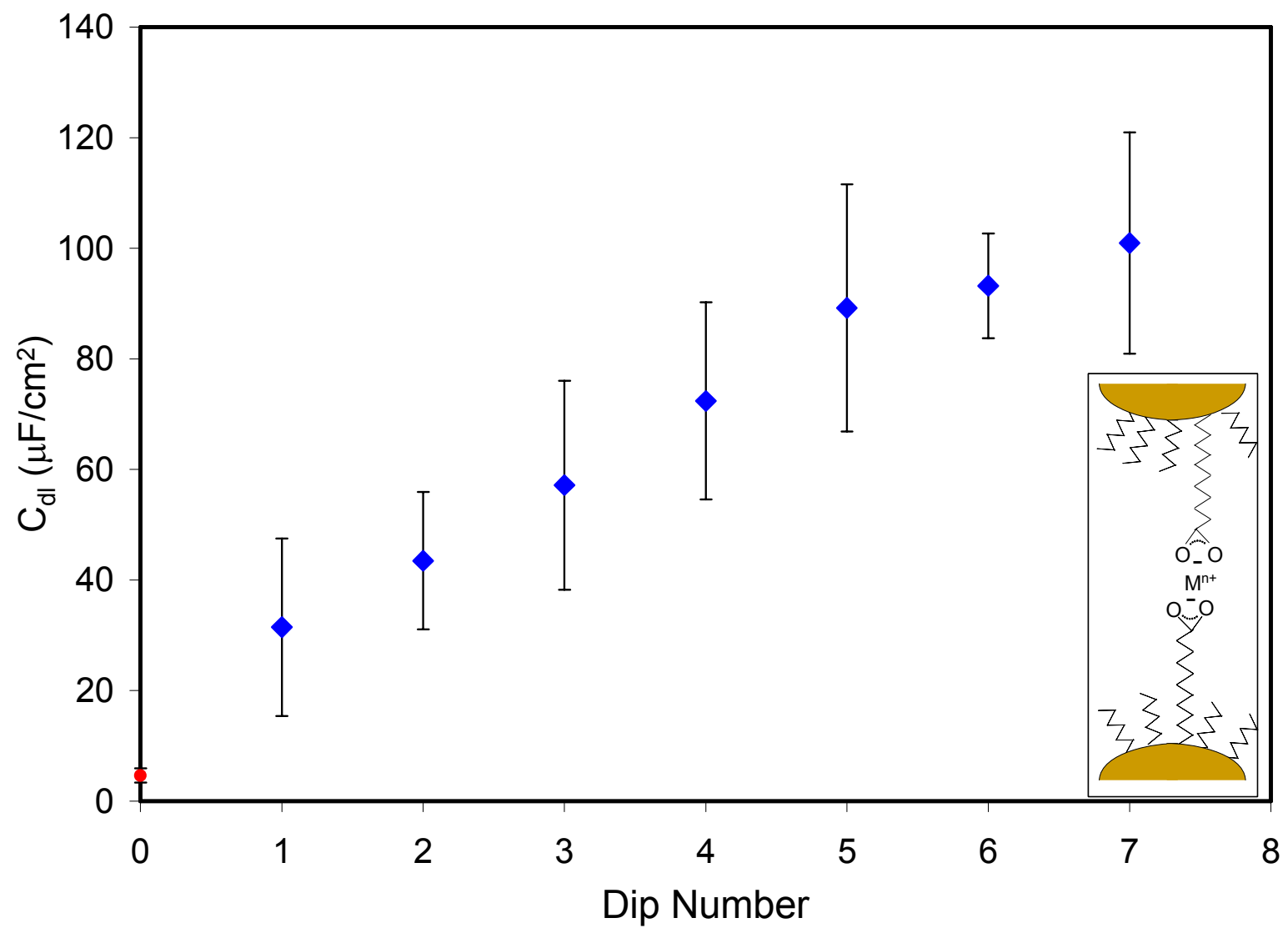

Figure SI-4. Double layer capacitance $\left(\mathrm{C}_{\mathrm{dl}}\right)$ of "metal-linked" (carboxylate-metal ion-carboxylate) MPC films. MPCs for these films are first place exchanged with MUA ligand in order to functionalize the nanoparticles with carboxylic acid terminated ligands that can be used in the linking bridges. The $\mathrm{C}_{\mathrm{dl}}$ of the initial MUA-SAM used to anchor the film is shown in red in the figure. The wide degree of variation in $\mathrm{C}_{\mathrm{dl}}$ with each exposure of the gold substrate to the MUA-MPC solution followed by immersion in metal ion solution, known as a "dip," is expected due to the presence of uncoordinated metal known to exist throughout the film.

Table SI-1. Contact Angle Measurements of Various Films on Gold

\begin{tabular}{l|c|c|c}
\hline \hline \multicolumn{1}{c|}{ System } & $\begin{array}{c}\text { Avg. Contact } \\
\text { Angle }\left(^{\circ}\right)\end{array}$ & $\begin{array}{c}\text { Standard } \\
\text { Deviation }\left(^{\circ}\right)\end{array}$ & n \\
\hline Decanethiol SAM & 92.6 & 1.9 & 3 \\
Octanethiol SAM & 103.4 & 2.8 & 3 \\
MPC Film (0 MUA) & 89.1 & 1.7 & 7 \\
MPC Film (21 MUA) & 81.7 & 2.4 & 3 \\
MPC Film (58 MUA) & 72.5 & 4.2 & 11 \\
MUA SAM & $21.5^{\dagger}$ & $--^{\dagger}$ & $1^{\dagger}$ \\
\hline
\end{tabular}

${ }^{\dagger}$ Literature values range from $20-25^{\circ} .{ }^{65}$ 


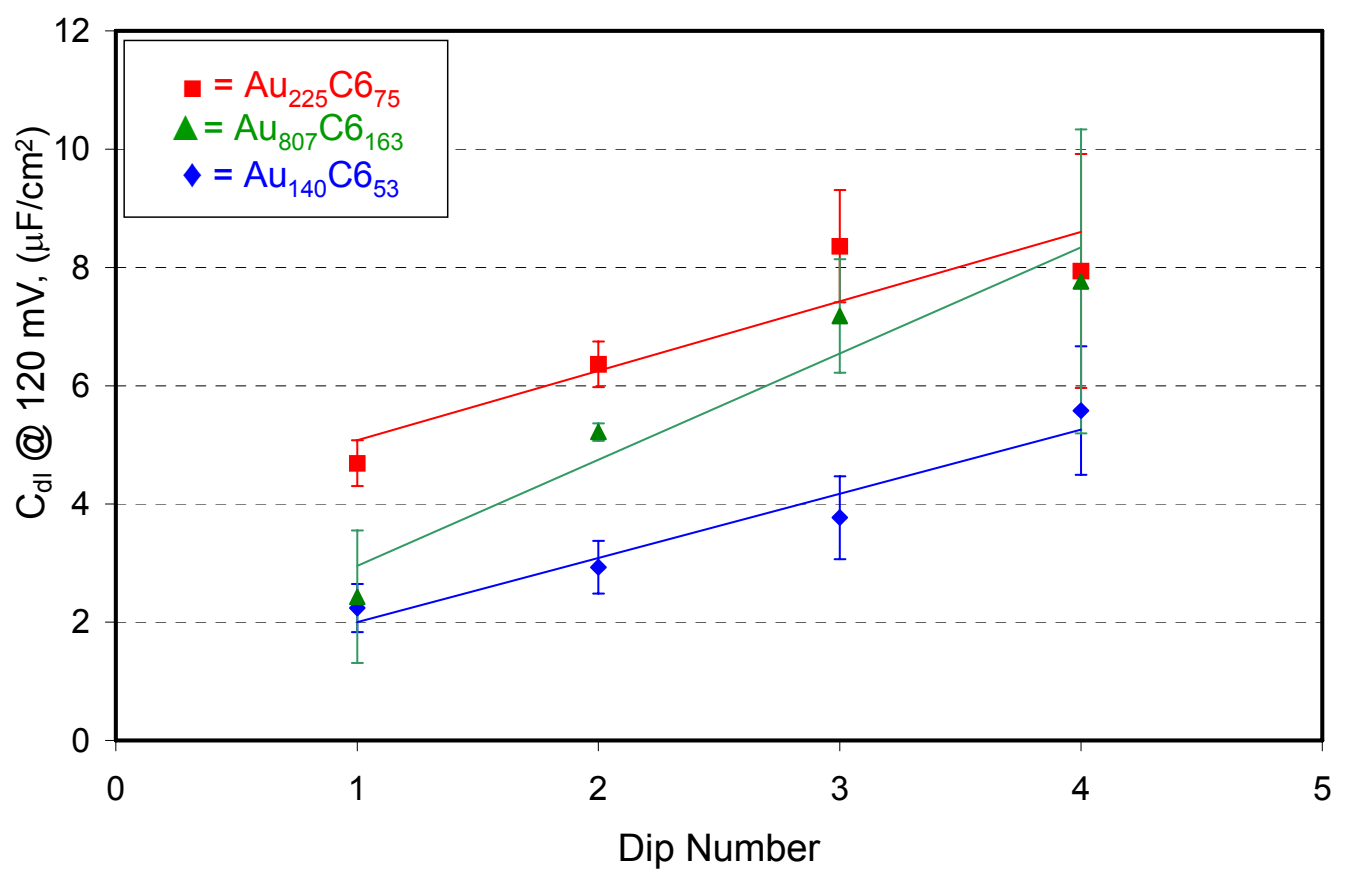

Figure SI-5. Double layer capacitance $\left(\mathrm{C}_{\mathrm{dl}}\right)$ of dithiol-linked MPC films assembled with MPCs of various core sizes. Films incorporating the largest cores, $\mathrm{Au}_{807} \mathrm{C}_{163}$, appear to have a trajectory for the highest capacitance as additional layers of MPC are added to the film. It can be speculated that because the larger cores have a smaller relative surface area composed of vertices and edges (illustrated below in Graphic 1, adapted from Landman et. $\mathrm{al}^{\mathrm{a}}$ ) and thus will have a peripheral ligand layer with a lower density of defects. This property, in turn, suggests the larger cores will interdigitate to a lesser degree than smaller particles, making packing order a more significant factor in overall film capacitance (i.e., packing of the larger particles may induce packing with more open space for solvent and electrolyte into the double layer, the consequence of which is an increased dielectric as well as a lower overall average distance between the outer Helmholtz plane and the electrode surface, both factors leading to higher capacitance.

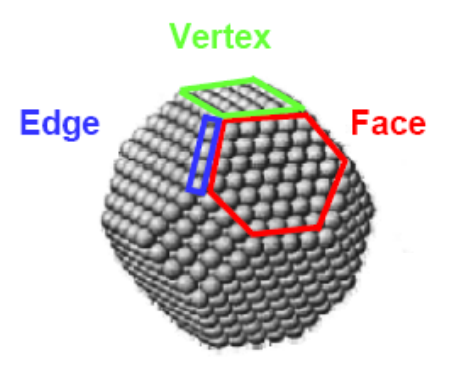

Graphic 1

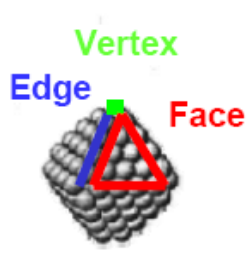

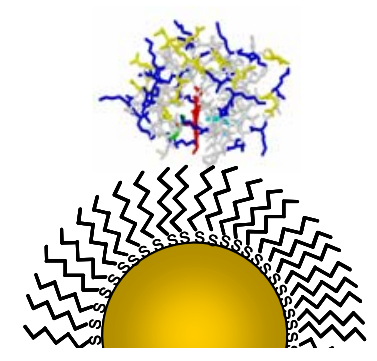

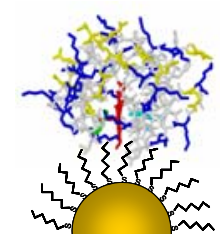

While there is a less substantial difference in the capacitance trends of the films composed of $\mathrm{Au}_{225}$ and $\mathrm{Au}_{140}$ nanoparticles, the higher surface coverage of edges and vertices in the smaller particles makes them more likely to expose adsorbed protein to the hydrophobic methylene chains of the ligands (Graphic 2). This interaction is suspected because we observed greater rates of cyt c denaturation upon adsorption to a $\mathrm{Au}_{140}$ interface (results not shown) compared to a $\mathrm{Au}_{225}$ interface (Figure 8). Thus, the $\mathrm{Au}_{225} \mathrm{MPCs}$ were selected for our MPC films assemblies because the offered the lowest capacitance trend coupled with a lower rate of denaturation. 


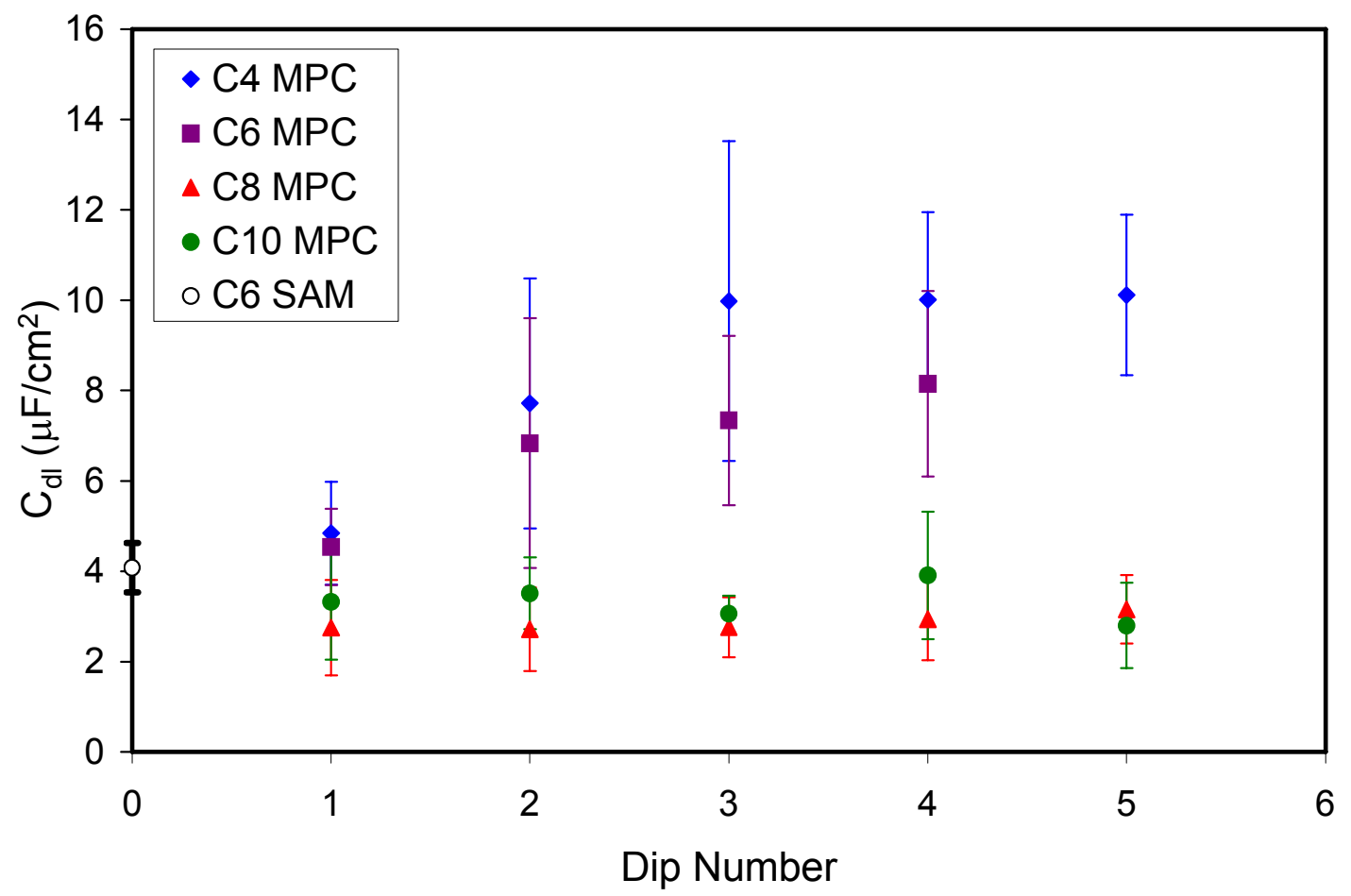

Figure SI-6. Double layer capacitance $\left(\mathrm{C}_{\mathrm{dl}}\right)$ of dithiol-linked MPC films assembled with $\mathrm{Au}_{225} \mathrm{MPCs}$ of various chainlength protecting ligands including butanethiolate (C4), hexanethiolate (C6), octanethiolate (C8), decanethiolate (C10), and dodecanethiolate (C12). The $\mathrm{C}_{\mathrm{dl}}$ trend for films of C12 MPCs is not shown since significant film growth was unachievable, most likely because the length of the protecting ligands (dodecanethiol) exceeds that of the linker ligand (nonanedithiol) making a linkage less probable. The $\mathrm{C}_{\mathrm{dl}}$ of the initial hexanethiol/nonanedithiol SAM (see Experimental Details) is shown as an open symbol at dip number 0. As seen in the figure, the film composed MPCs with longer chainlength ligands (C10 and C8) display a low capacitance over the five dip cycles shown. Film growth with these materials, however, is inefficient as the length of the protecting ligands partially inhibits the dithiol connections between nanoparticles. The films of C4 MPCs grow much more efficiently but are the least effective at insulating the polar cores in terms of capacitance. Moreover, C4 MPCs, with a thinner protective layer, are more likely to aggregate over time. From our experiments, it was determined that the C6 MPCs offer the greatest advantages in terms of stability, linking efficiency, and an acceptable level of film capacitance. It should be noted from the figure that many of the films, especially those with longer chainlength protection, exhibited a lower $\mathrm{C}_{\mathrm{dl}}$ with the initial layer of MPC (dip 1). As illustrated below in Graphic 3, this observation is reasonable due to the very low dielectric (highly non-polar) environment created when the first layer of MPCs is attached to the SAM modified substrate.
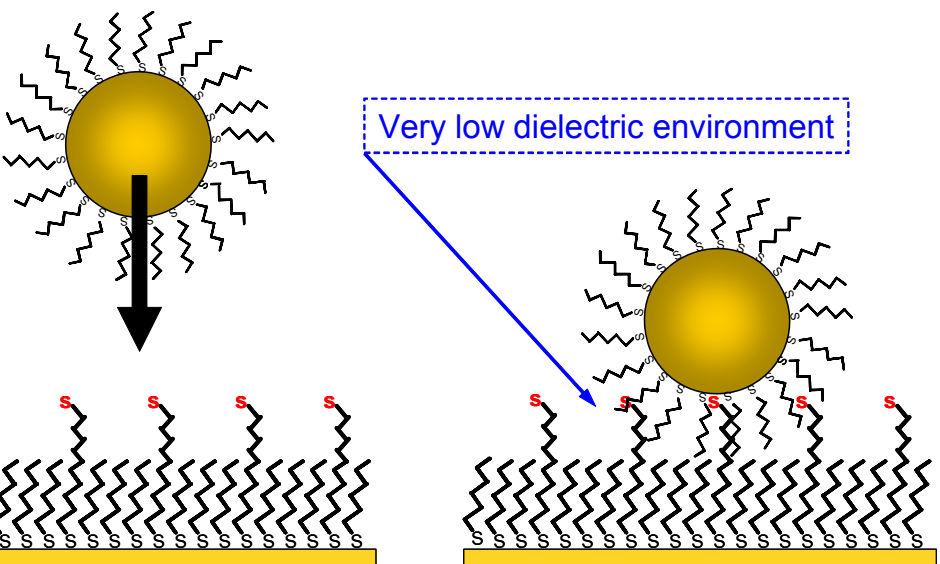

Graphic 3

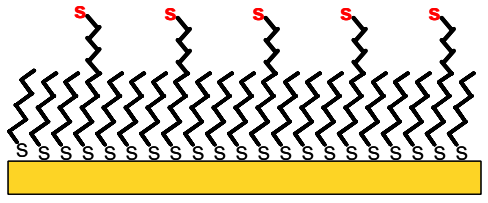




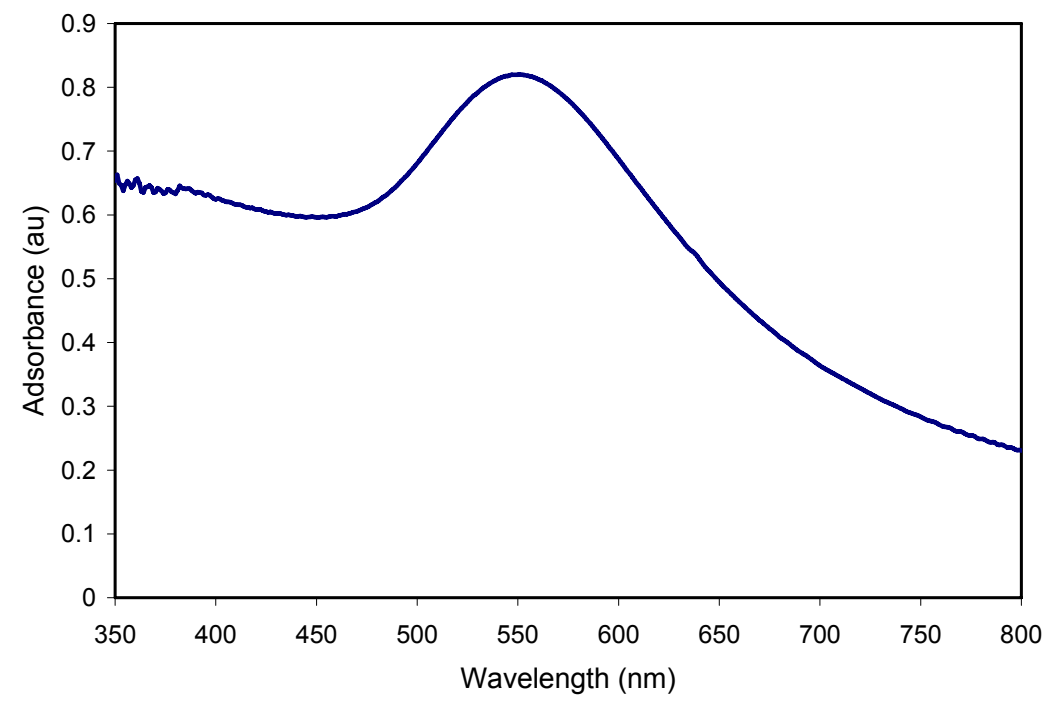

Figure SI-7. UV-Vis spectrum of as-prepared MUA-capped MPCs synthesized with an adaptation of the Rubinstein procedure.

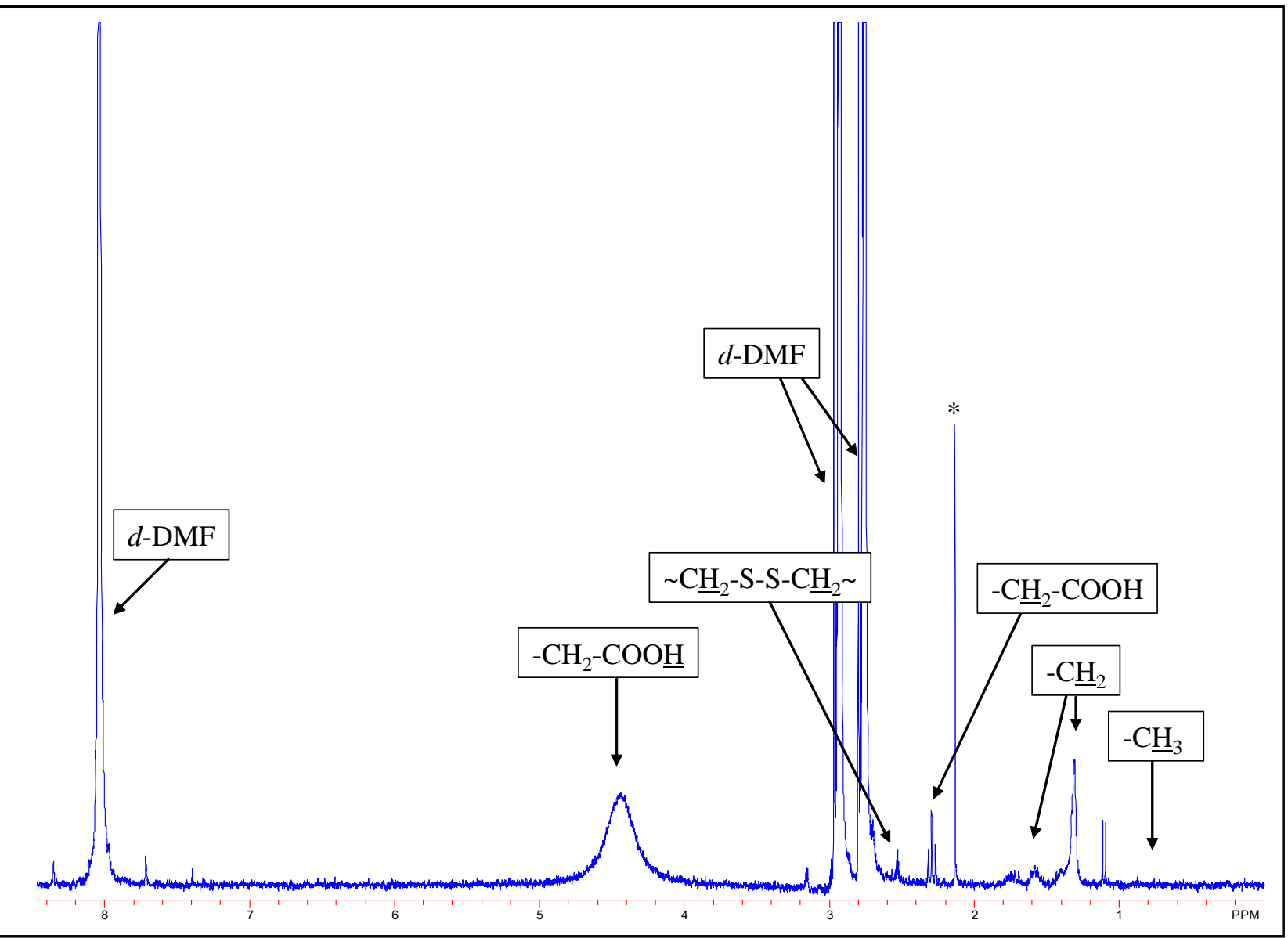

Figure SI-8. ${ }^{1} \mathrm{H}$ NMR of iodine-decomposed sample of as prepared MUA-MPCs. Note: * indicates residual signal from the acetone used in the preparation process. The lack of a signal resulting from protons on methyl groups, coupled with the strong MUA signal indicates successful creation of the MUAcapped MPCs. 

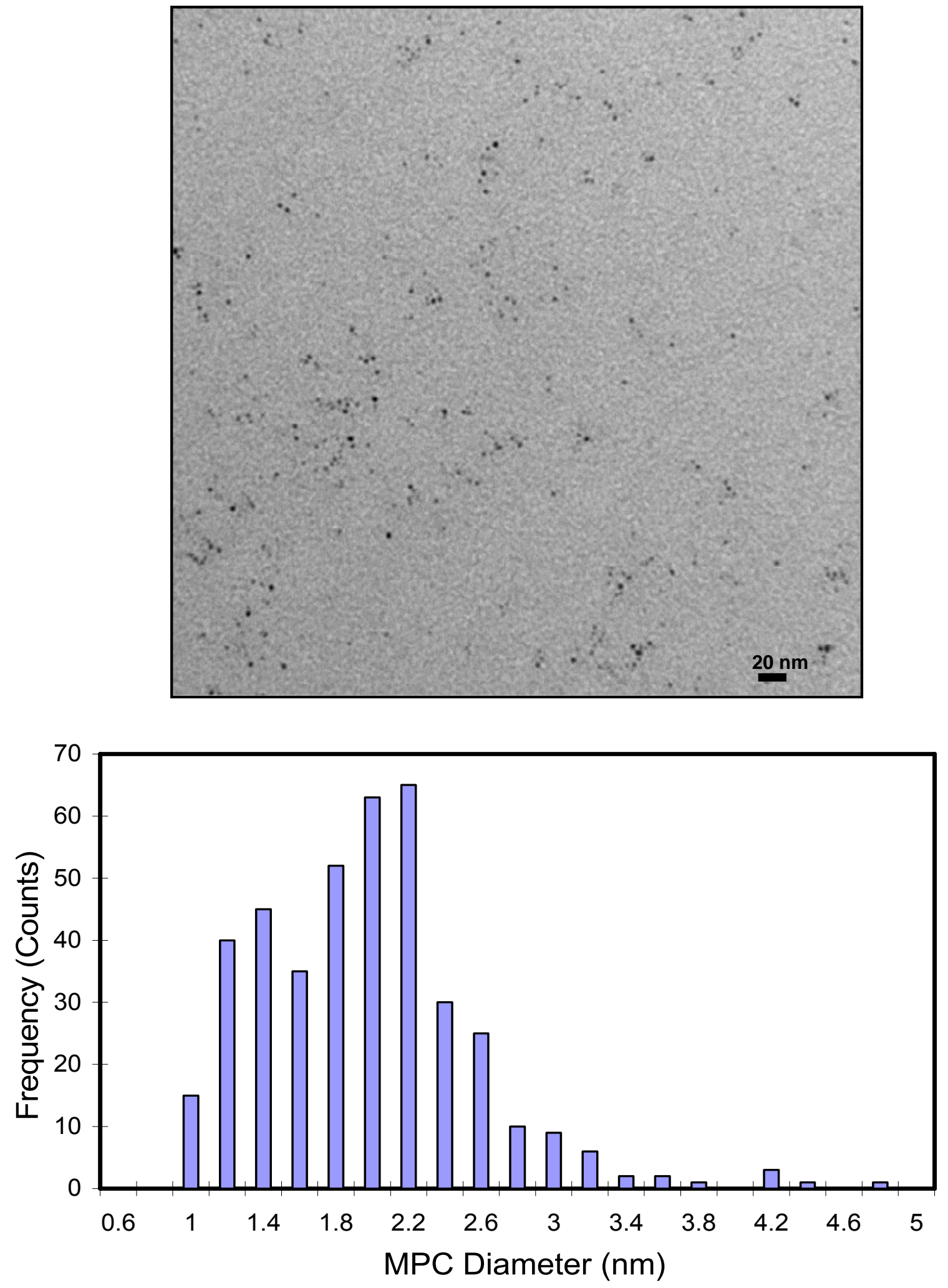

Figure SI-9. Typical TEM image (top) of hydrophilic MUA-capped MPCs with an core size histogram (bottom) from image analysis of multiple TEM images of as prepared MUA-MPCs indicating an average diameter of $1.86 \pm 0.59 \mathbf{~ n m}$. 

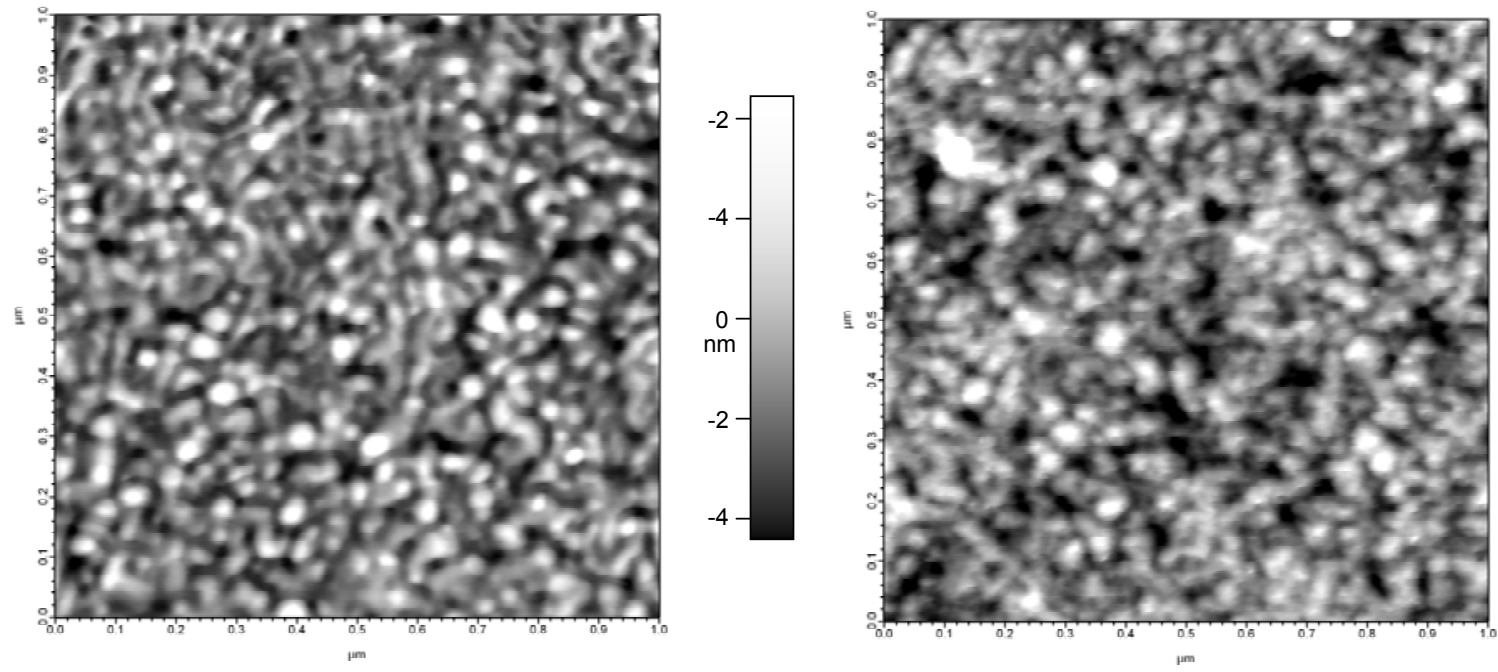

Figure SI-10. “Tapping mode” atomic force microscopy images $(1 \mu \mathrm{m} \times 1 \mu \mathrm{m})$ of the bare evaporated gold substrate (left) and with an five layer, dithiol-linked MPC film assembly (right). The RMS roughness of each substrate was calculated as $1.7_{7} \mathrm{~nm}$ and $1.8_{8} \mathrm{~nm}$, essentially the same and in excellent agreement with previous in lab measurements and literature reports. ${ }^{b}$ For comparison, hydrogen flame annealed evaporated gold and gold epitaxially grown on mica were determined to have RMS roughness factor of $\sim 0.9 \mathrm{~nm}$ and 0.3, respectively (Asylum Atomic Force Microscope; silicon nitride cantilever).

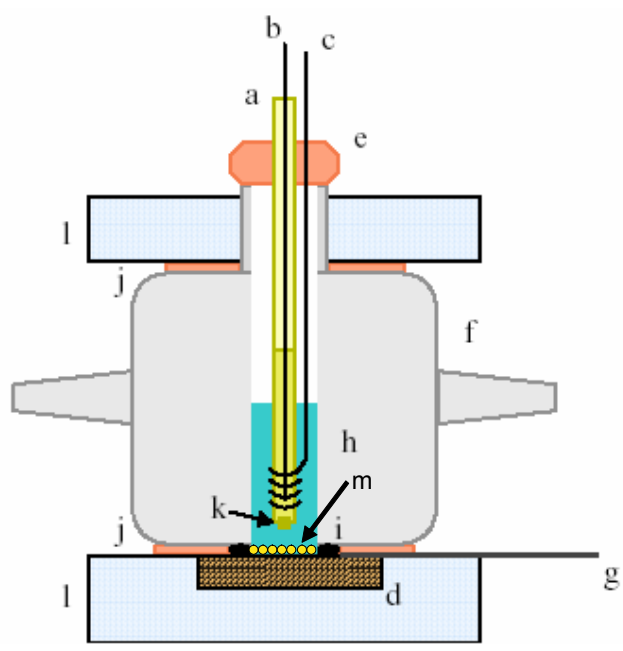

Figure SI-11. Schematic illustration of the electrochemical cell used in this research: a) reference electrode housing (glass barrel with $1 \mathrm{M} \mathrm{KCl}$ ), b) $\mathrm{Ag} / \mathrm{AgCl}$ reference wire, c) a Pt auxiliary electrode wire, d) SAM modified gold working electrode, e) rubber stopper fitted to hold both the glass reference barrel and the Pt counter electrode, f) glass cell body, g) brass electrical contact to gold working electrode, h) buffered electrolyte solution, i) Viton o-ring, j) rubber gaskets, $\mathrm{k}$ ) a glass frit acting as a salt bridge, l) Lucite retainer plates, m) MPC film assembly. The entire cell is held together with threaded rods and wing nuts (not shown).

\section{Additional References}

${ }^{a}$ W.D. Luedtke and U.J. Landman J. Phys. Chem. B 1998, 102, 6566-6572.

${ }^{\mathrm{b}}$ C.R. Clemmer and T.P. Beebe Scanning Microscopy 1992, 6(2), 319-333. 